\title{
A Novel method for license plate location
}

\author{
Jiawen Song ${ }^{1, \mathrm{a}}$, Chuang $\mathrm{Zhu}^{2, \mathrm{~b}}$, Xiaodong $\mathrm{Xie}^{2, \mathrm{c}, *}$, Yuan $\mathrm{Li}^{2, \mathrm{~d}}$,Meng Wang ${ }^{2, \mathrm{e}}$, and Huizhu Jia ${ }^{2, \mathrm{f}}$ \\ ${ }^{1}$ School of Electronics Engineering And Computer Science, Peking University, 100871, Beijing, China \\ ${ }^{2}$ National Engineering Laboratory of Video Technology, Peking University, China \\ a jiawens@pku.edu.cn, ${ }^{\mathrm{b}}$ czhu@pku.edu.cn, ${ }^{\mathrm{c}}$ donxie@pku.edu.cn, ${ }^{\mathrm{d}}$ yuanli@pku.edu.cn, ${ }^{\mathrm{e}}$ \\ mengw@pku.edu.cn, ${ }^{\mathrm{f}}$ hzjia@pku.edu.cn
}

Keywords: license plate, Sobel filter, hsv colour space.

\begin{abstract}
With license plate recognition technology, police can better manage vehicle and this technique also give convenience to application such as parking fee charge. In this paper, we propose a novel method to locate license plate of china in complicate environment. First, we change the RGB image into greyscale image. Then, we apply Sobel operator on the grey-scale image to get its Sobel edge image. After that the Sobel edge image is change into a binary image in which each pixels of the image are decided by statistic its variance in nearby vertical and horizontal pixels. Base on the binary image above, the algorithm locates the candidate boxes and produces a list for them. Next, we select the boxes above by using hsv colour space and then merge the boxes base on their location. At last, the unreasonable boxes are removed and the final location of license plates produced. Our method has outperformed two types of license plate location products in a small surveillance dataset and achieved a fast and high precision location result compared with the-state-of-art.
\end{abstract}

\section{Introduction}

Automatic license plate recognition(ALPR) is an important system designed for vehicle management. In recent year, numerous real-life applications take license plate recognition as key technique, such as toll collection system, parking fee charge and road monitoring. License plate location is the first step in ALPR and plays an important role in it. The precision of license plate location influences the final result of recognition directly. In real world, license plate location faces many problems. These problems are summarized as follows. First, plate variations in location, quantity, camera, colour, font, standard versus vanity, occlusion, inclination and other. Secondly, environment variations include illumination and background. The variations mentioned make license plate location become a difficult problem.

In the past decades, researchers have tried to solve this problem with different methods to get better result. [1]-[4] used edge detection methods to locate license plate and they tried to locate the license plate by finding all possible rectangular shape. In [5]-[8], Sobel filter is used to detect edges. Then two vertical lines and two horizontal lines are detected to locate the edge of license plate. Because license plate in different countries always have specific colour, [9] proposed a method to locate license plate by classify all the pixels in the image using the hue, lightness, and saturation (HLS) colour model. In [10]-[12],adaptive boosting is used to detect license plate by equipping Haar-like features. Methods in [10]-[12] tries to detect license plate by locating text feature on it.

The methods mentioned above can get good result on some dataset. But in real-life application, the environment is complicated. To evaluate the performance of an algorithm, we should get a harder dataset to check in. In this paper, we propose a new method to locate license plate in China in a faster and more precision way. The rest of this paper includes three section. In section two, we will illustrate the structure of our method, its advantages compared with the-state-of-art, details of every part in our method and our experiment result compared with two kinds of products in a small but harder surveillance dataset. In section three, we have concluded this paper and our contribution. 


\section{Structure of the proposed method}

In this section, we will first illustrate the main structure of our method and offer the intermediate results in 2.1. Then, details of each steps are shown in section2.2. In section 2.3, environment of our experiment and results compare with two types of license plate recognition products are shown.

\subsection{Eight steps of the whole structure}

Our method combines texture feature and colour feature to get a fast and precision result in complicated environment. As figure 1 shown, our method can be divided into eight parts. First, $11 * 11$ median filtering is performed to the input image to eliminate noise of the image which will affect our edge detection result. Secondly, the produced image is transformed to HSV colour space to find out the hue value nearest the range of blue colour. Meanwhile, the produced image in first step is also transformed into grey-scale image and then Sobel filter is applied on the grey-scale image to get edge response image. Thirdly, a binary image is produced based on the hue and local response variance. Then we apply broad first search to find out boxes of connected pixels on binary image. Next, the candidate boxes are selected by their area. After merging small boxes by their location, the final step is to select the correct boxes by their ratio of length to width.

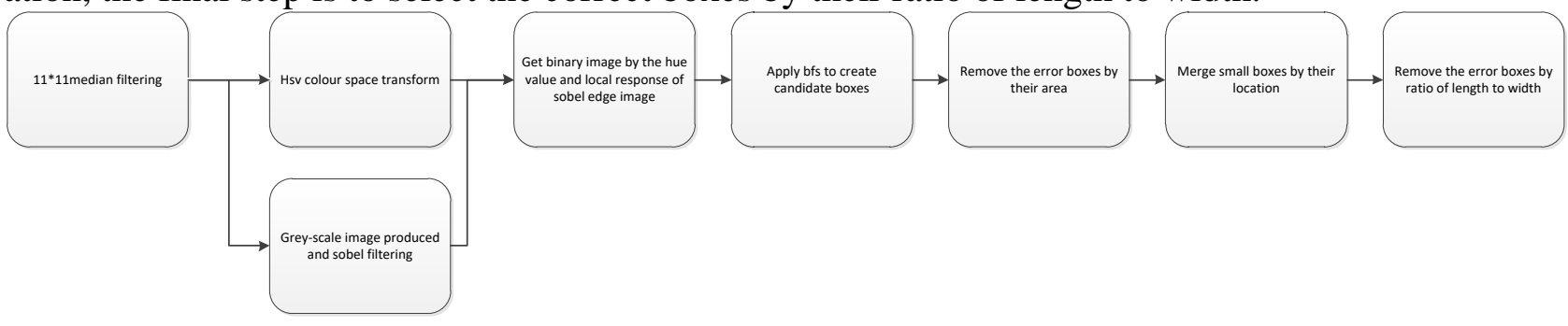

Figure 1 Structure of our method

The middle results of first two steps are shown in figure 2. Noise of the input image is depressed obviously in median filter image. And in hsv colour space image, the three channel is change into hue, saturation and value. The hsv colour space is more similar to human vision, so we produce the hsv colour space image to make use of the information of hue. The grey-scale image is produced to maintain texture information which is prepared for Sobel edge image.
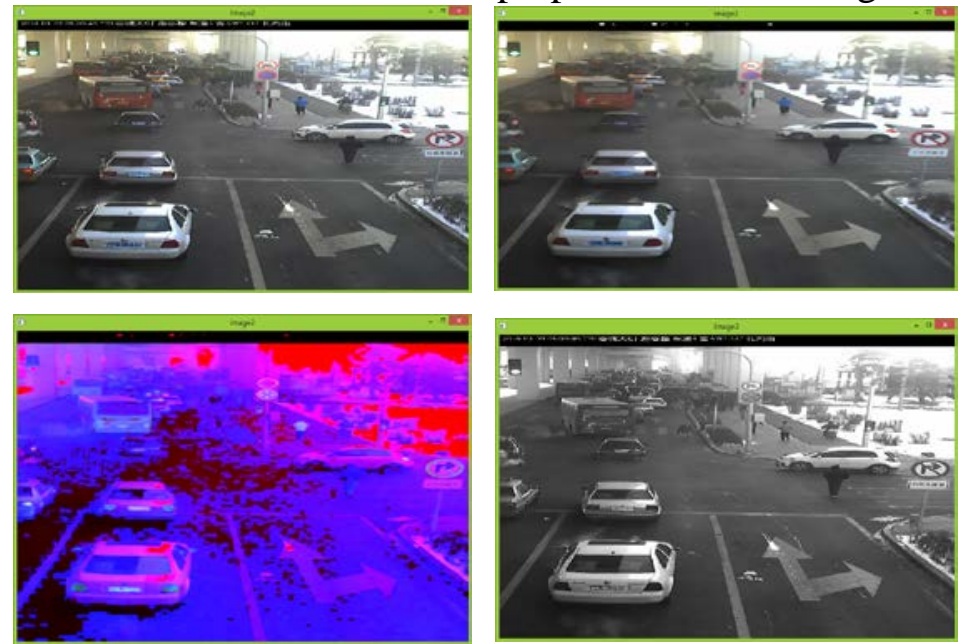

Figure 2 intermediate result of the algorithm. Top left is the input image, top right is median filter image, down left is hsv colour space image and down right is grey-scale image.

In figure 3, the result of the rest steps is shown. Top left is edge image produce by apply Sobel filter on grey-scale image. It has maintained the edge of grey-scale image and outlined the profile of object such as car and text on the license plate. Then the binary image is produced by hsv image and Sobel edge image. The binary image is shown on top right and the procedure of its computation is detailed in section 2.2 . Candidate boxes is produced by using broad first search(bfs) on binary image. Bfs takes pixels adjacency as a candidate box and produces a candidate boxes list. Down left is the candidate boxes list after first selection which has removed boxes with abnormal area. Down 
middle is the result after merge the adjacent boxes. Down right is the final boxes list after second selection. License plate contain more than 25 pixels are detected.

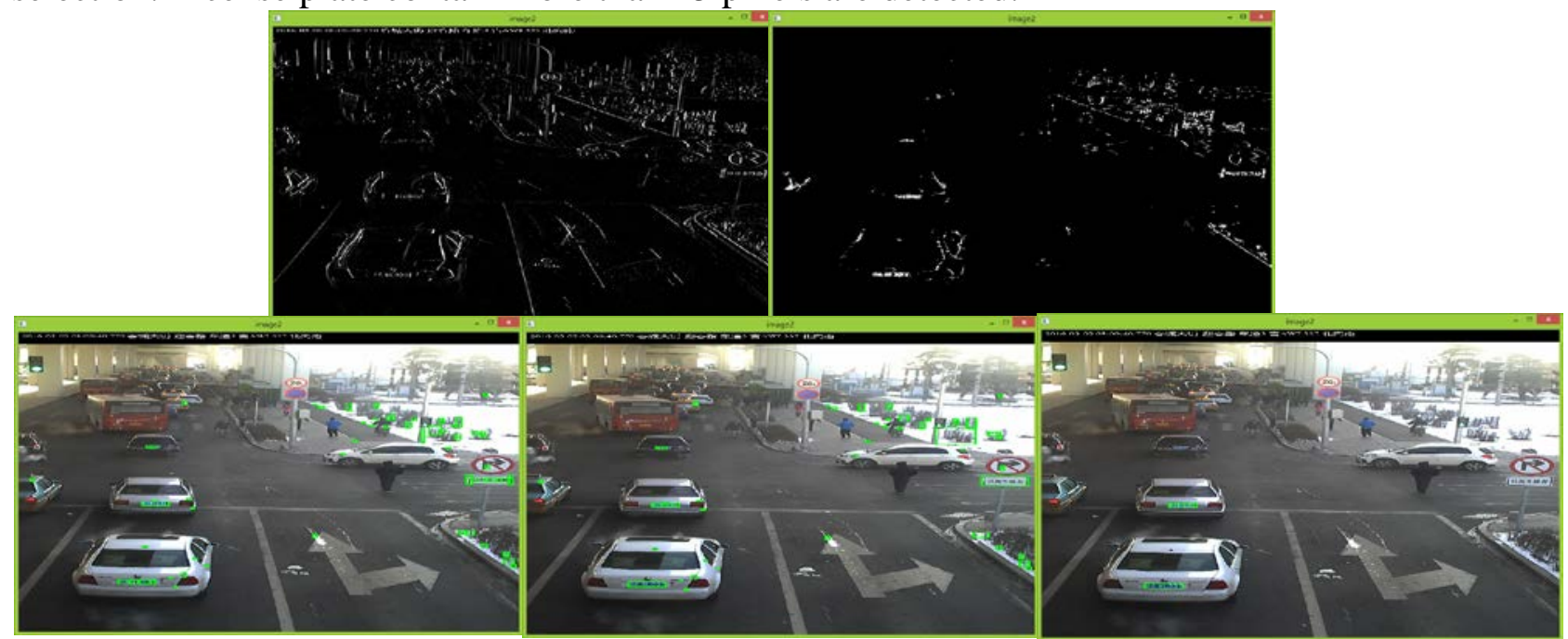

Figure 3 intermediate result of the rest. Top left is the Sobel edge image, top right is binary image, down left shows candidate boxes after first selection, down middle shows result of merge and down right is the final result.

\subsection{Detail of binary and twice boxes selection}

In this part, procedure of binary image transform is formularized. Because of the texture on license plate arranged horizontally, the gradient of pixels in these areas changes with higher frequency than smooth areas on Sobel edge image. To utilize the characteristic of texture on license plate, we statistic every pixel in Sobel edge image. Each pixel is represented with two response values of their variance in a horizontal window and a vertical window. The two response value is defined as follow:

$$
\begin{array}{r}
\text { vresponse }=\mathrm{D}\left(p_{y-2}, p_{y-1}, p_{y}, p_{y+1}, p_{y+2}\right) \\
\text { hresponse }=\mathrm{D}\left(p_{x-4}, p_{x-3}, p_{x-2}, p_{x-1}, p_{x}, p_{x+1}, p_{x+2}, p_{x+3}, p_{x+4}\right)
\end{array}
$$

vresponse represents the response value on vertical direction and hresponse represents the response value on horizontal direction. The window size on vertical direction is defined to 5 while horizontal's is defined to 9. This setting is defined to suit size of Chinese character.

Usage of hsv colour space image is devided into two steps. The first step is to find out the hue in the image which is closest to standard blue. This step is try to find out the most blue point in the image which is influence by complicated environment such as illumination. And then we have a base blue hue of the hsv colour image. At last, every point of the image binary by the follow formula:

$\operatorname{pixel}_{x, y}=\left\{\begin{array}{l}0, \text { vresponse }>\text { vthreshold, hresponse }>\text { hthreshold, }\left|h_{x, y}-h_{s}\right|<\text { huethreshold } \\ 1, \text { vresponse } \leq \text { vthreshold, hresponse } \leq \text { hthreshold, }\left|h_{x, y}-h_{s}\right| \geq \text { huethreshold }\end{array}\right.$

pixel $_{x, y}$ represent the pixel value in binary image with coordinate $\mathrm{x}$ and $\mathrm{y} . h_{s}$ represents hue of the nearest standard blue detected in hsv colour image. Vthreshold represents threshold of vertical response and hthreshold represents the horizontal one. Huethreshold is applied to limit the distance between hue value of binary pixel and hue value of the nearest standard blue. The procedure of binary is simplified in figure 4 . 


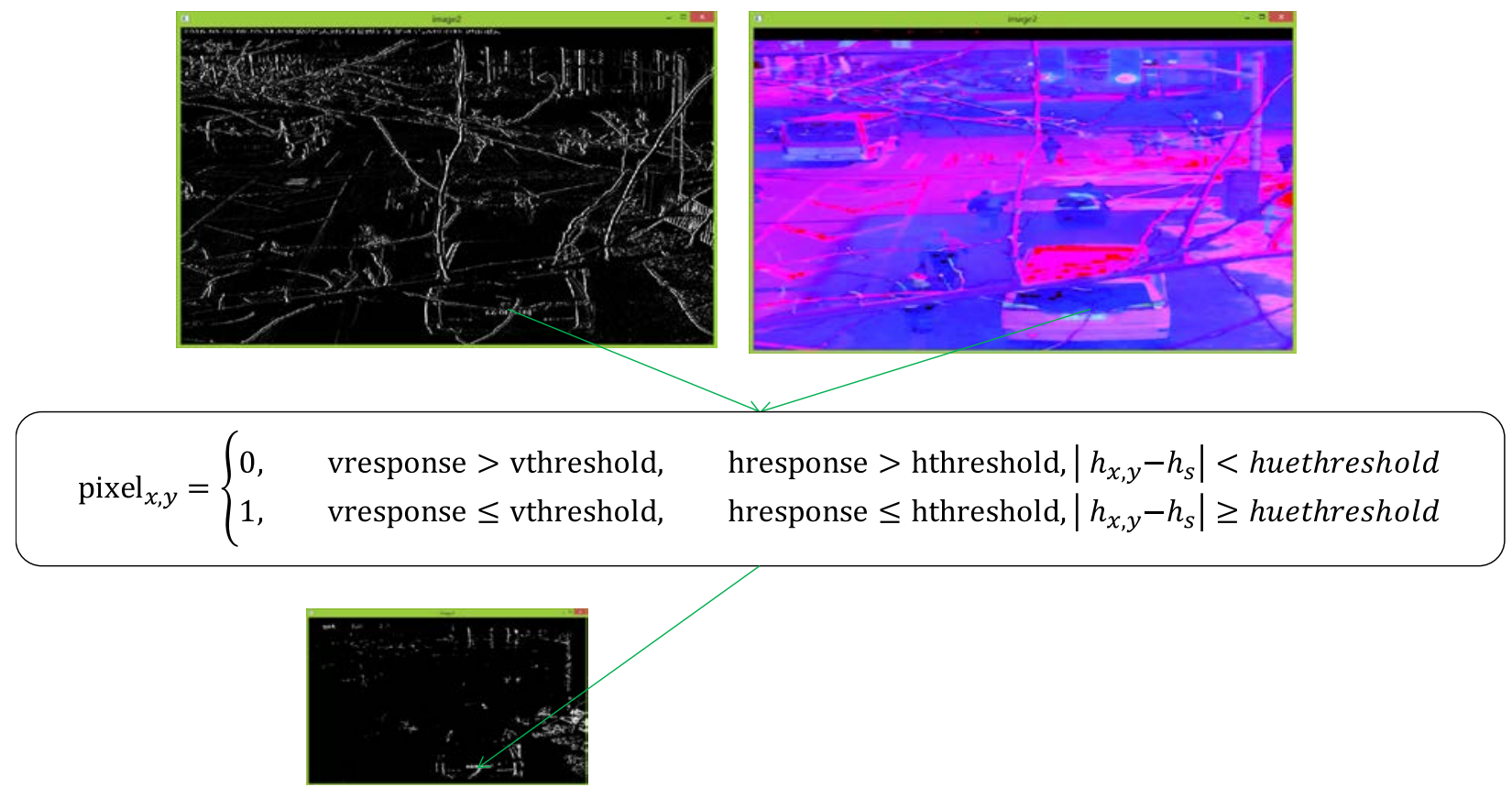

Figure 4 procedure of binary.

After binary image produced, bfs is applied to produced candidate boxes. Then, first selection of the boxes list is performed. Since license plate location is to locate license plate in median size, if a candidate box is too small or too big it has a high probability to be an error candidate box. So in the first selection, candidate boxes with large area or little area are removed. And the first selection of candidate boxes can also avoid error in box merge step. If the large or small boxes remained to merge, the boxes caused by noise or some similar areas will mix up with the correct box which results in a worse result. An example of first selection is shown in figure 5.

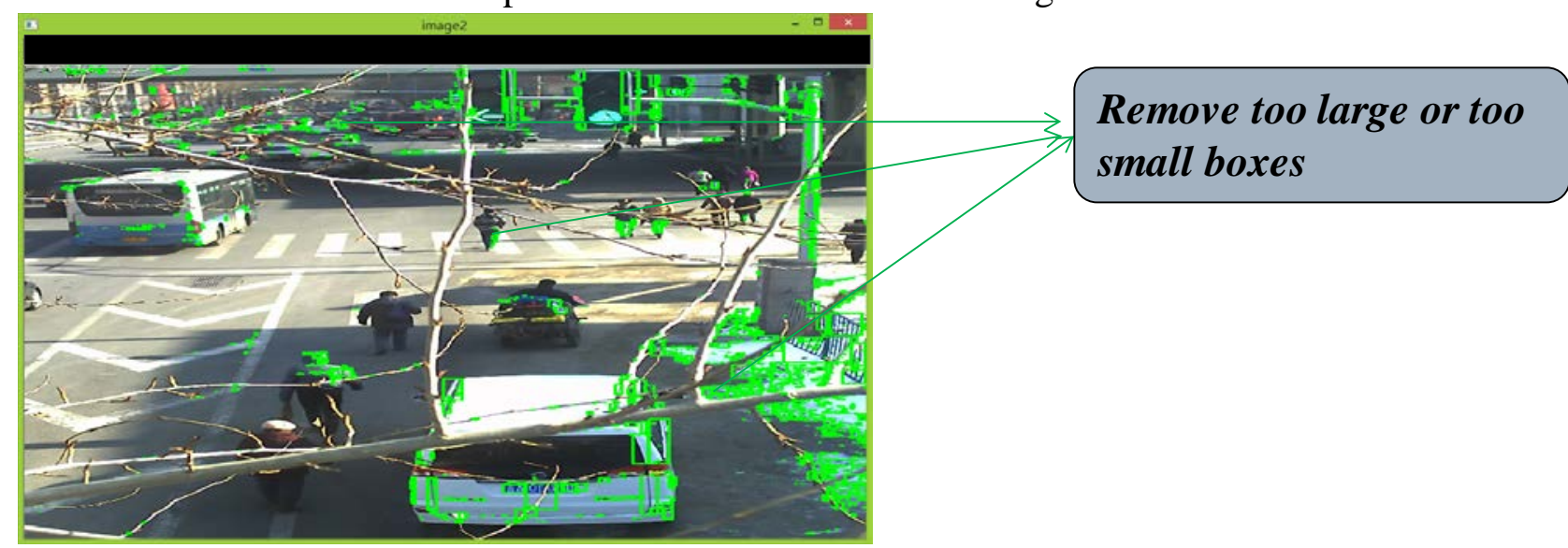

Figure 5 first selection of candidate boxes.

In the merge step, boxes is merged base on their location. This step aims to merge the area broken by occlusion or blur caused by complicated environment. The recover bounding boxes contains the correct bounding boxes of license plate. Merge operation of two boxes is decided by their relation value I which is computed as formula 4 shown. overlap $v$ represents the overlap pixel number of two boxes vertically. $d i s_{H}$ represents distance between boxes horizontally while w and h represent width and height of them. If I larger than threshold, boxes will be merge into one.

$$
\mathrm{I}=\frac{\text { overlap }_{V}}{\min \left(h_{1}, h_{2}\right)}-\frac{\text { dis }_{H}}{\sqrt{w_{1 w_{2}}}}
$$

After merge step, there are still some error boxes remain in our list. Compared with these boxes, license plate bounding boxes have some different characteristics. Utilizing these special property, we can filter out the final result. The second selection of the boxes illustrated in figure 6 . 


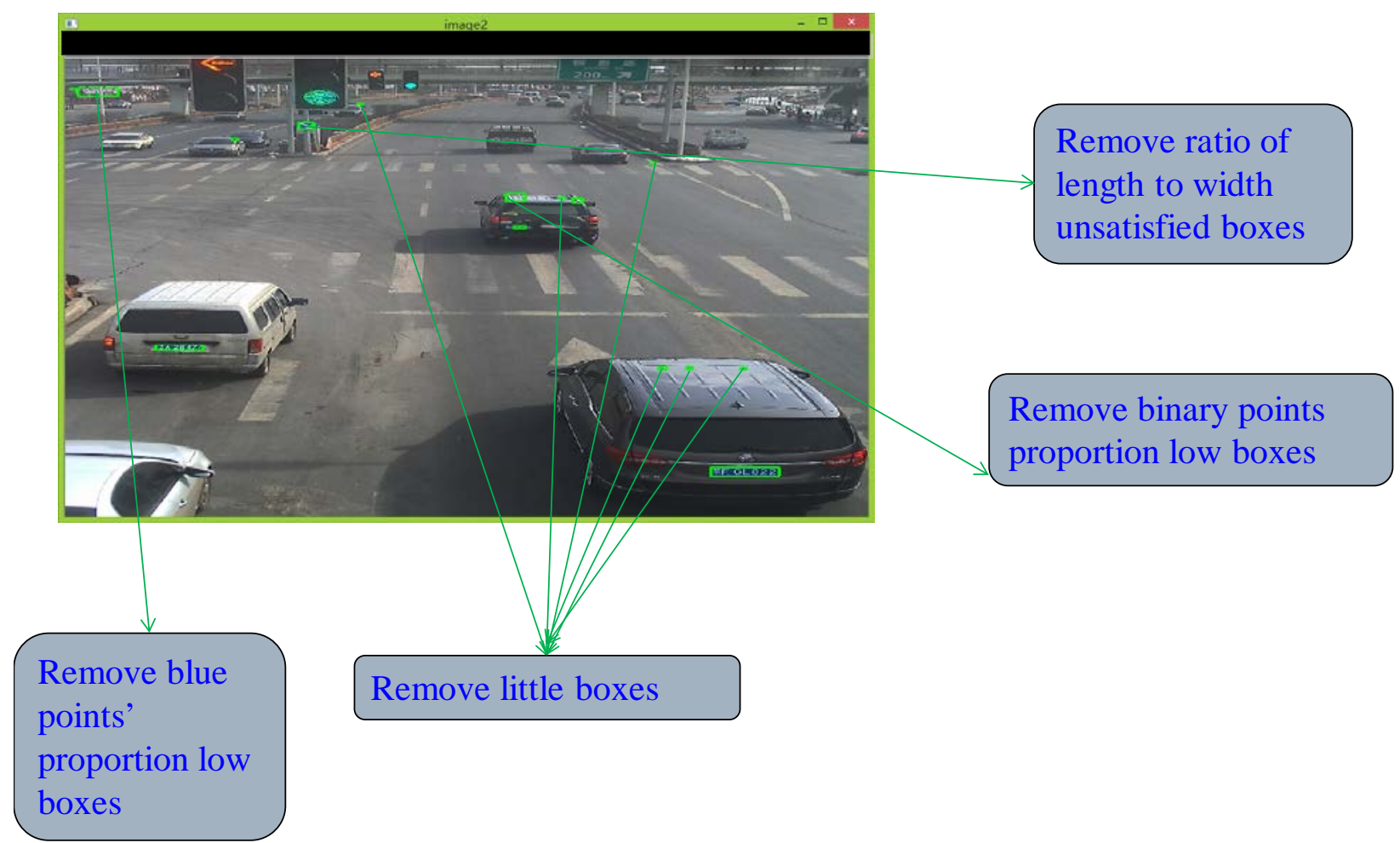

Figure 6 second selection of candidate boxes

Four types of boxes will be removed after second selection. The small boxes with little pixels will be removed because they are not the suitable size. Boxes with ratio of length to width far from license plate are removed, too. The proportion of binary points contained in a candidate box is considered as the third condition to select the correct box, because boxes with low proportion means that they lack of texture and blue points. The final condition is the proportion of blue points of the box. A point in image can be easily defined to blue point or not by using the detected hue of standard blue mentioned above. At last, the remain boxes will be considered as the correct bounding boxes of license plate.

\subsection{Environment setting, dataset description and experiment result}

Our experiment is performed on a machine with Intel core i5-4258u cpu and 4GB ram. The dataset contains 1000 images which are took from real world surveillance video. The dataset is much harder than dataset used for research. In this dataset, our algorithm has outperformed two types of license plate recognition product. The result of the experiment is shown in table 1.

Table 1 Experiment result

\begin{tabular}{|c|c|c|}
\hline algorithm & Error rate & precision \\
\hline huoyanzhenjing & $5.6 \%$ & $91.3 \%$ \\
\hline wentong & $6.5 \%$ & $82.5 \%$ \\
\hline Proposed method & $5 \%$ & $93 \%$ \\
\hline
\end{tabular}

\section{Conclusion}

In this paper, we have proposed a novel method for license plate location. The proposed method constructs a complete procedure for license plate location and get a robust result in low computation. Our method combines texture and colour feature of license plate and achieves a better result compared with two types of license plate recognition product. 


\section{Acknowledgements}

National Key Research and Development Program of China under contract No. 2016YFB0401904

\section{References}

[1] H. Bai and C. Liu, "A hybrid license plate extraction method based on edge statistics and morphology,” in Proc. Int. Conf. Pattern Recognit., vol. 2. 2004, pp. 831-834.

[2] D. Zheng, Y. Zhao, and J. Wang, “An efficient method of license plate location,” Pattern Recognit. Lett., vol. 26, no. 15, pp. 2431-2438, 2005.

[3] S. Wang and H. Lee, "Detection and recognition of license plate characters with different appearances,” in Proc. Int. Conf. Intell. Transp. Syst., vol. 2. 2003, pp. 979-984.

[4] F. Faradji, A. H. Rezaie, and M. Ziaratban, “A morphological-based license plate location,” in Proc. IEEE Int. Conf. Image Process., vol 1. Sep.-Oct. 2007, pp. 57-60.

[5] M. Sarfraz, M. J. Ahmed, and S. A. Ghazi, "Saudi Arabian license plate recognition system,” in Proc. Int. Conf. Geom. Model. Graph., 2003, pp. 36-41.

[6] D. Zheng, Y. Zhao, and J. Wang, “An efficient method of license plate location,” Pattern Recognit. Lett., vol. 26, no. 15, pp. 2431-2438, 2005.

[7] K. Kanayama, Y. Fujikawa, K. Fujimoto, and M. Horino, "Development of vehicle-license number recognition system using real-time image processing and its application to travel-time measurement,” in Proc. IEEE Veh. Tech. Conf., May 1991, pp. 798-804.

[8] S. Zhang, M. Zhang, and X. Ye, "Car plate character extraction under complicated environment," in Proc. IEEE Int. Conf. Syst. Man Cybern., vol. 5. Oct. 2004, pp. 4722-4726.

[9] X. Shi, W. Zhao, and Y. Shen, “Automatic license plate recognition system based on color image processing,” Lecture Notes Comput. Sci., vol. 3483, pp. 1159-1168, 2005.

[10] H. Zhang, W. Jia, X. He, and Q. Wu, "Learning-based license plate detection using global and local features,” in Proc. Int. Conf. Pattern Recognit., vol. 2. 2006, pp. 1102-1105.

[11] W. Le and S. Li, “A hybrid license plate extraction method for complex scenes,” in Proc. Int. Conf. Pattern Recognit., vol. 2. 2006, pp. 324-327.

[12] L. Dlagnekov, License Plate Detection Using AdaBoost. San Diego, CA: Computer Science and Engineering Dept., 2004 